\title{
Providers' views on PrEP for adolescent girls and young women in Tanzania: Findings from implementation science research
}

Population Council

Follow this and additional works at: https://knowledgecommons.popcouncil.org/departments_sbsr-hiv

Part of the Demography, Population, and Ecology Commons, Family, Life Course, and Society Commons, International Public Health Commons, and the Medicine and Health Commons How does access to this work benefit you? Let us know!

\section{Recommended Citation}

Population Council. 2017. "Providers' views on PrEP for adolescent girls and young women in Tanzania: Findings from implementation science research," DREAMS Tanzania Results Brief 2. Washington, DC: Population Council. 


\section{PROVIDERS' VIEWS ON PREP FOR ADOLESCENT GIRLS AND YOUNG WOMEN IN TANZANIA}

Adolescent girls and young women (AGYW) continue to have high rates of HIV in Tanzania, where HIV prevalence triples from 1.3 percent among adolescent girls aged $15-19$ to 4.4 percent among young women aged $20-24 .{ }^{1}$ AGYW in Tanzania, as in many countries, are particularly vulnerable to HIV due to a host of reasons, including economic disadvantage and limited power to negotiate condom use or refuse sex with their male partners. ${ }^{2}$ Until recently, interventions to reduce HIV within this population focused only on addressing knowledge, attitudes, and behaviors, with limited success. ${ }^{2}$ Biomedical interventions that AGYW can access and use to protect themselves from HIV have been lacking.

Oral pre-exposure prophylaxis (PrEP), which are antiretroviral medications taken by HIV-uninfected persons to prevent HIV, has the potential to substantially reduce HIV acquisition among AGYW as part of a comprehensive HIV prevention package, if they are able to access, use, and adhere to it. ${ }^{3-6}$ The World Health Organization recommends ${ }^{7}$ that PrEP should be offered as an prevention choice for people at substantial risk for HIV infection; for AGYW, this includes being in sero-discordant or transactional sex relationships, experiencing gender-based violence, or sexually transmitted infections (STIs). ${ }^{2}$

Health care providers (HCPs) are critical for effective provision of quality PrEP services for AGYW. ${ }^{8}$ However, this requires substantial commitment on their part since HCPs are responsible for conducting a clinical assessment of the potential PrEP user, prescribing PrEP, and providing specialized counseling, routine HIV testing, and close, long term monitoring for side effects. ${ }^{9}$ As policymakers, programmers, and other stakeholders consider PrEP for AGYW in Tanzania, it is important to understand HCPs' perceptions of PrEP and gauge their willingness to prescribe it to AGYW in order to inform the PrEP implementation process and to make it a useful tool against HIV.

In collaboration with the National AIDS Control Programme (NACP) and CSK Research Solutions Ltd, the Population Council conducted an implementation science research study in Tanzania to identify key considerations among HCPs for PrEP introduction to AGYW in an urban and a rural setting in

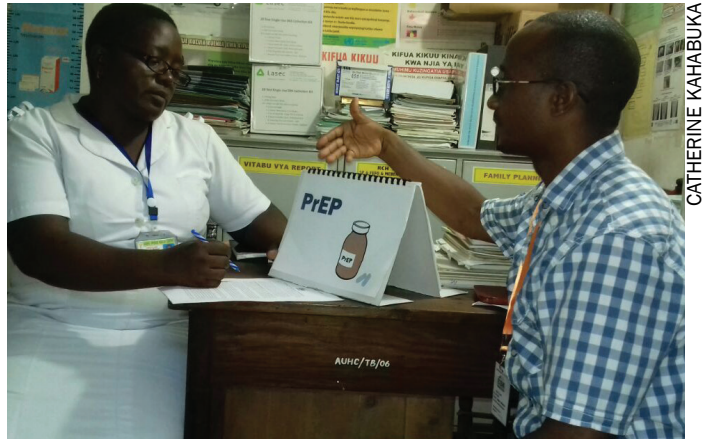

It is important to understand health care providers' perceptions of PrEP and gauge their willingness to prescribe it to AGYW.

\section{KEY FINDINGS}

Although only 3 percent of HCPs had prior knowledge of PrEP, most HCPs were in favor of providing PrEP to AGYW at high risk of HIV once informed about the PrEP.

HCPs' biases toward AGYW sexuality may influence whether, and if so, how they prescribe PrEP to AGYW.

HCPs require support on working with AGYW as well as on values clarification to address potential biases toward AGYW sexuality.

Existing health facility procedures may influence PrEP prescribing behaviors of HCPs; training of HCPs to administer PrEP should include components on improving the facilities' quality of care.

HCPs support different cadres of HCPs administering PrEP to AGYW as well as PrEP integration into general sexual and reproductive health services.
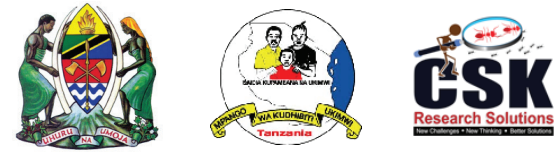
Dar Es Salaam and Mbeya districts, respectively. In this brief, we present results from a survey and indepth interviews (IDIs) conducted with HCPs in each setting, assessing their:

- Knowledge, attitudes and perceptions about PrEP.

- Willingness to prescribe PrEP to AGYW.

- Perceived barriers and facilitators to providing PrEP to AGYW.

\section{METHODS}

HCPs who provide sexual and reproductive health services in 74 randomly selected clinics completed a detailed questionnaire from March to April 2017. ${ }^{10}$ Trained research assistants administered the survey questions on HCP characteristics and current PrEP knowledge. Following these questions, the research assistants used a standardized script to provide information on PrEP to all HCPs. Placebo PrEP pills were also shown to the HCPs. Thereafter, the HCPs completed the remaining sections of the survey on their own. Additionally, 24 HCPs were selected from the survey sample to participate in IDIs focused on understanding their attitudes and perceptions towards PrEP and facilitators and barriers to providing PrEP, especially to AGYW. The study was approved by the Population Council Institutional Review Board (New York, USA) and the National Institute of Medical Research (Dar es Salaam, Tanzania).

\section{RESULTS}

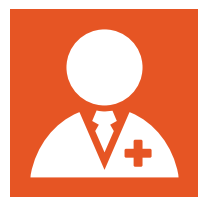

\section{Who participated in the} study?

A total of 316 HCPs completed the survey; 196 (62 percent) from 35 health facilities in Dar es Salaam and 120 (38 percent) from 39 health facilities in Mbeya. Surveyed HCPs were from dispensaries (58 percent), hospitals (21 percent) and health centers (21 percent). Most HCPs worked in public-managed facilities (61 percent). The mean age of the sample was 40 years, most were female and about half were nurses (Table 1). The Dar es Salaam sample was older, had more females, and more years working in their profession than the Mbeya sample.

Only 3 percent of providers had prior knowledge of PrEP. Most HCPs reported caring for adolescents younger than age 18, although the proportion was significantly higher for HCPs in Mbeya compared to Dar es Salaam.

A total of $24 \mathrm{HCPs}, 12$ in each district, participated in the IDIs. An equal proportion (38 percent) of the sample worked in health centers and dispensaries and the majority worked in public facilities (63 percent). The mean age of the providers was 41 years. The majority were female (63 percent) and nurses (42 percent).

TABLE 1 HCPS' DEMOGRAPHIC CHARACTERISTICS

\begin{tabular}{|c|c|c|c|c|}
\hline & \multirow{2}{*}{$\begin{array}{c}\text { IDIs } \\
(n=24) \\
\%\end{array}$} & \multicolumn{3}{|c|}{ Quantitative surveys } \\
\hline & & $\begin{array}{c}\text { Dar es Salaam } \\
\mathrm{n}=196 \\
\%\end{array}$ & $\begin{array}{c}\text { Mbeya } \\
n=120 \\
\%\end{array}$ & $\begin{array}{c}\text { Total } \\
\mathrm{n}=316 \\
\%\end{array}$ \\
\hline Age $(\text { mean })^{* * *}$ & 41.3 & 42.1 & 37.3 & 40.3 \\
\hline Female sex ${ }^{* *}$ & 62.5 & 77.0 & 61.7 & 71.2 \\
\hline $\begin{array}{l}\text { Main profession }{ }^{\star * *} \\
\text { Nurse } \\
\text { Doctor } \\
\text { Clinical officer } \\
\text { Counselor } \\
\text { Other }\end{array}$ & $\begin{array}{c}41.7 \\
25.0 \\
16 ., 7 \\
- \\
16.7\end{array}$ & $\begin{array}{r}52.6 \\
19.4 \\
6.1 \\
19.9 \\
1.3\end{array}$ & $\begin{array}{r}42.5 \\
8.3 \\
23.3 \\
1.7 \\
9.5\end{array}$ & $\begin{array}{l}48.7 \\
15.2 \\
12.7 \\
13.0 \\
10.8\end{array}$ \\
\hline Year worked in profession* (mean) & - & 14.7 & 11.0 & 13.3 \\
\hline Provide care to clients under age $18^{* * *}$ & - & 83.6 & 96.7 & 87.3 \\
\hline Prior knowledge of PrEP & - & 4.6 & 1.7 & 3.5 \\
\hline
\end{tabular}

${ }^{* * *}<0.001 ;{ }^{* *}<0.01 ;{ }^{*}<0.05$ 


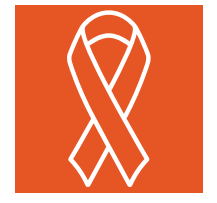

How willing are HCPs to provide PrEP to AGYW subpopulations at high risk of HIV?

Greater than 90 percent of HCPs were willing to provide PrEP to AGYW in sero-discordant relationships, AGYW who exchange sex for money, and AGYW who change partners frequently (Figure 1). Fewer HCPs were willing to provide PrEP to AGYW with a history of abuse from partners or STIs. Only 61 percent of providers were willing to prescribe PrEP to all AGYW at high risk for HIV. During the qualitative interviews, HCPs explained that AGYW were in need of PrEP due to sexual violence (e.g., rape) and engagement in unprotected sex.

\section{This young generation will benefit because they are in a risky environment, traumatized, raped and forced to get married when they are very young. The use of PrEP will prevent[them] from getting HIV infection.}

-Male clinical officer, age 27 , public dispensary

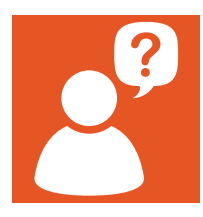

What are HCPs' main concerns about prescribing PrEP?

Across the two settings, the three main concerns about PrEP, reported

by over 30 percent of providers, were concerns about increased high risk behavior, side effects, and patient noncompliance with therapy (Figure 2).

\section{FIGURE 1 HCPS' WILLING TO PRESCRIBE PREP BY AGYW SUB-POPULATION}

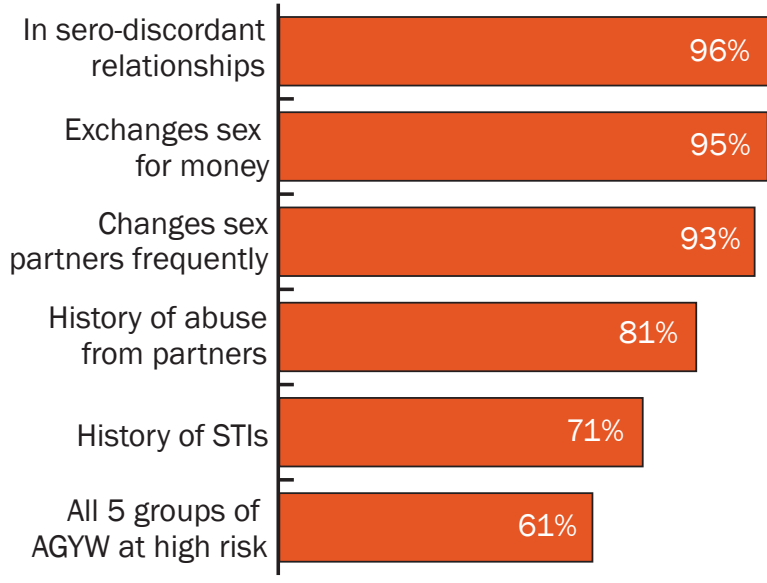

FIGURE 2 HCPS' MAIN CONCERNS ABOUT PREP

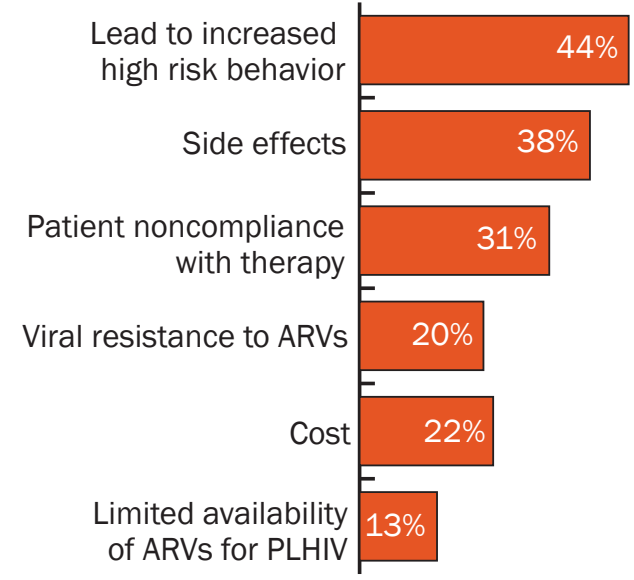

While these concerns were echoed during the indepth interviews, HCPs noted that proper education and counselling to clients could address concerns around proper use and dealing with side effects, and prioritizing safer sexual behaviours. As one provider stated:

\section{Some people might think that they are free to do whatever they want [due to PrEP use] as they are assured of not getting HIV infection. So, we should provide education and advise them on using [PrEP]".}

-Female nurse, age 34, public dispensar

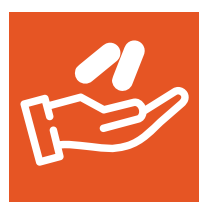

\section{Where, how, and by whom should PrEP be provided for AGYW?}

Across the two settings, HCPs were more supportive of PrEP being offered through public and private health facilities compared to nongovernmental organizations, mobile health services and faith-based organizations (Figure 3).

HCPs also suggested that PrEP services could be integrated into existing maternal and reproductive health (87 percent) and youth-friendly health services (92 percent), such as, family planning (91 percent), STI prevention and treatment (88 percent), and gender-based violence counseling (82 percent). Some interviewed HCPs explained that currently antiretrovirals (ARVs) are provided at HIV care and 
FIGURE 3 HCPS' VIEWS ON SERVICE LOCATIONS TO PROVIDE PREP TO AGYW

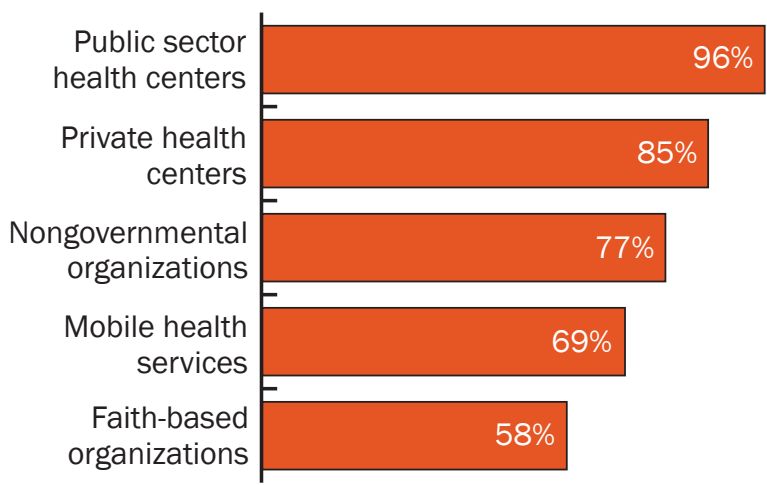

treatment centers and anyone seen entering these centers are assumed to be living with HIV. Therefore, PrEP should not be offered at these centers but integrated with other services to minimize potential stigma.

\begin{abstract}
14 The best environment [to provide PrEP] should be multi-sectoral...As you know, girls and young women tend to shy off in public clinics which are involved in providing HIV services; therefore, this will provide more privacy. As I said before if the services [referring to PrEP] are made multi-sectoral, then privacy will have been created.
\end{abstract}

-Male clinical officer, age 32, public hospital

During the survey, HCPs wanted trained medical professionals to provide PrEP. In addition to doctors, more than 85 percent of HCPs supported PrEP provision by nurses, counselors, and clinical officers. Only 65 percent of HCPs supported pharmacists prescribing PrEP. In the IDIs, however, they recognized that PrEP uptake might be most effective if the AGYW were reached by those in the community who could advise and counsel potential clients.

Interviewer:

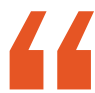

Okay can you tell me why do you think CBHs [Community-based health workers] will be the best rank of service providers to provide PrEP to the youth?
Respondent:

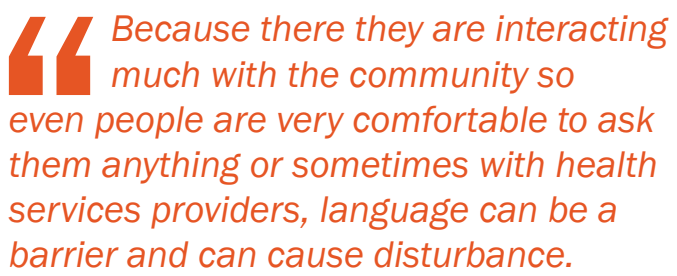

-Female nurse, age 34, public dispensary

\section{Are there urban and rural differences in factors associated with providers' willingness to prescribe PrEP to all AGYW at high risk of HIV?}

There were significant differences by setting on the factors influencing providers' willingness to prescribe PrEP to AGYW at high risk for HIV (Table 2).

In Mbeya, HCPs with greater concerns that PrEP would result in increased risk behavior reported being less willing to prescribe PrEP to AGYW in comparison to HCPs with fewer concerns.

In Dar es Salaam, HCPs with more negative attitudes toward AGYW's sexuality and with greater concerns about how PrEP will be integrated into existing services reported being less willing to prescribe PrEP to AGYW in comparison to HCPs with fewer negative attitudes and fewer concerns, respectively. Some interviewed HCPs explained that HCPs are apprehensive about providing sexual and reproductive health services to unmarried AGYW. Cultural beliefs about pre-marital sexual activity often results in unmarried girls who are sexually active being stigmatized by their communities, including HCPs, as promiscuous.

\footnotetext{
4 Those who are not married, there is a bit of stigma to some of them, they [referring to HCPs] feel that when they start using contraceptives they will be having bad sexual behaviors. Even some of the service providers have stigma especially those who are young. Because in the culture of our society that when someone talks about contraceptives she should be married, if not married she is a
} 
TABLE 2 FACTORS ASSOCIATED WITH HCPS' WILLINGNESS TO PRESCRIBE PREP TO AGYW AT HIGH RISK FOR HIV

\begin{tabular}{|c|c|c|c|c|}
\hline & \multicolumn{2}{|c|}{$\begin{array}{l}\text { Dar es Salaam } \\
\qquad(n=196)\end{array}$} & \multicolumn{2}{|c|}{$\begin{array}{c}\text { Mbeya } \\
(n=120)\end{array}$} \\
\hline & $\begin{array}{c}\% \\
\text { willing }\end{array}$ & p-value & $\begin{array}{c}\% \\
\text { willing }\end{array}$ & p-value \\
\hline $\begin{array}{l}\text { Fear of increased risk behavior } \\
\text { Few } \\
\text { Many }\end{array}$ & $\begin{array}{l}60.0 \\
57.3\end{array}$ & ns & $\begin{array}{l}82.5 \\
49.2\end{array}$ & $<0.001$ \\
\hline $\begin{array}{l}\text { Concerns about PrEP integration into services }{ }^{\text {b }} \\
\text { Low } \\
\text { High }\end{array}$ & $\begin{array}{l}70.0 \\
53.1\end{array}$ & $<0.05$ & $\begin{array}{l}72.9 \\
54.0\end{array}$ & ns \\
\hline $\begin{array}{l}\text { Negative attitudes toward AGYW sexualityc } \\
\text { Few } \\
\text { Many }\end{array}$ & $\begin{array}{l}71.3 \\
48.6\end{array}$ & $<0.01$ & $\begin{array}{l}72.1 \\
55.8\end{array}$ & ns \\
\hline
\end{tabular}

Note: significance applies only to survey sample

a5 items (e.g., PrEP will cause patients to engage in riskier behaviors)

${ }^{\mathrm{b}} 4$ items (e.g., I do not have time to provide clinical monitoring for PrEP)

'11 items (e.g., Unmarried AGYW should not be provided contraceptives because Tanzanian culture does not support premarital sex)

strange person. Therefore, customs and traditions enhance the stigma as well.

-Male doctor, age 57, public health center

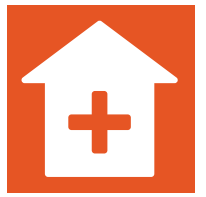

Are there urban and rural differences in how current service facility procedures influence HCPs' readiness to prescribe PrEP to all AGYW at high risk of HIV?

In both districts, HCPs who reported that their facility had high quality patient-centered care practices were more willing to prescribe PrEP to AGYW that those who reported their facility had low quality patient-centered practices (Table 3).

There were also significant differences by setting regarding health facility factors. In Mbeya, HCPs who report that their facility has appropriate guidelines for working with adolescents were more willing to prescribe PrEP to AGYW than those who reported their facilities did not have appropriate guidelines.

In Dar es Salaam, HCPs who reported that providers in their facility were sufficiently trained to provide

TABLE 3 ASSOCIATIONS BETWEEN HCPS' PERCEPTIONS OF HEALTH FACILITY SERVICES AND WILLINGNESS TO PRESCRIBE PREP TO AGYW AT HIGH RISK FOR HIV

\begin{tabular}{|c|c|c|c|c|}
\hline & \multicolumn{2}{|c|}{$\begin{array}{l}\text { Dar es Salaam } \\
\quad(n=196)\end{array}$} & \multicolumn{2}{|c|}{$\begin{array}{c}\text { Mbeya } \\
(n=120)\end{array}$} \\
\hline & $\%$ willing & p-value & $\%$ willing & p-value \\
\hline $\begin{array}{l}\text { Patient-centered care practices } \\
\text { High } \\
\text { Low }\end{array}$ & $\begin{array}{l}67.8 \\
54.7\end{array}$ & $<0.05$ & $\begin{array}{l}85.0 \\
55.0\end{array}$ & $<0.01$ \\
\hline $\begin{array}{l}\text { Has guidelines for working with adolescents } \\
\text { Yes } \\
\text { No }\end{array}$ & $\begin{array}{l}59.0 \\
57.6\end{array}$ & ns & $\begin{array}{l}71.2 \\
52.3\end{array}$ & $<0.05$ \\
\hline $\begin{array}{l}\text { HCPs trained to provide HIV services to adolescents } \\
\text { Yes } \\
\text { No }\end{array}$ & $\begin{array}{l}73.5 \\
55.6\end{array}$ & $<0.05$ & $\begin{array}{l}81.3 \\
62.5\end{array}$ & ns \\
\hline
\end{tabular}

a4 items (e.g., Providers take time to understand clients' needs) 
HIV prevention, care, and treatment services to adolescents and young adults were more willing to prescribe PrEP to AGYW than HCPs who reported that providers were insufficiently trained.

Interviewed HCPs in both districts described clinical environments that would discourage AGYW's use and adherence to PrEP. They noted that adolescents are often criticized and judged by some of their colleagues and that there was a lack of respect and confidentiality when providing care to adolescents.

\begin{abstract}
4 [Providers] just judge someone.., "ooh you came again? last time you had gonorrhea, did you do it again?" I have experienced many of these behaviors...there was a woman who somehow missed that appointment, when she showed up another day she was welcomed with harsh words and insults. Now this patient is on daily medication [referring to PrEP] which is not even friendly to her, and you have to give her counseling to believe what she is taking is not actually medications but something to help her. So the problem is that most of us are critical and judgmental.
\end{abstract}

-Male assistant clinical officer, age 32 , public dispensary

HCPs described needing continuous, rigorous onthe-job training in order to improve the quality of care provided to young women.

\section{Although they are all trained personnel some may neglect a few things and they need to get refresher course training in certain areas.}

-Male clinical officer, age 27, public dispensary

\section{HCP CURRICULUM THAT WORKS}

The Council developed a training curriculum for health care settings that has been shown to reduce provider stigma and bias toward young people at risk of HIV and increase the quality of services provided to young people. ${ }^{11}$ The curriculum includes sessions on values clarification, talking about sex, understanding gender, and providers being the change to remove stigma and biases. Curriculums like this one can be adapted as part of the PrEP implementation strategy for AGYW in Tanzania.

\section{RECOMMENDATIONS}

Our findings highlight key considerations that should be addressed as part of PrEP introduction to AGYW in Tanzania. We recommend the following:

1. Address HCPs' views on adolescent sexuality by implementing comprehensive and continuous training on caring and working with adolescents and young people that includes, values clarification exercises (see box for example HCP curriculum).

2. Address differences in willingness to prescribe PrEP by assessing HCPs' perspectives periodically, and providing guidelines and training in HIV service delivery.

3. When implementing PrEP in urban and rural locations, pay careful attention to geographical differences in quality of care provided by facilities, provider attitudes, and training needs.

4. Reach larger segments of AGYW who are at-risk of HIV by integrating PrEP into general sexual and reproductive health services and by developing guidelines and tools to facilitate integration.

5. Engage HCPs at all stages of PrEP roll-out, from planning to introduction to evaluation. 


\section{REFERENCES}

${ }^{1}$ Tanzania Commission for AIDS (TACAIDS) et al. 2013 Tanzania HIV/AIDS and Malaria Indicator Survey 2011-12. Dar es Salaam, Tanzania:

TACAIDS, ZAC, NBS, OCGS, and ICF International.

${ }^{2}$ Celum, C. L.. et al. 2015. "Rethinking HIV

prevention to prepare for oral PrEP implementation

for young African women," Journal of the

International AIDS Society 18(Suppl 3): 20227.

${ }^{3}$ WHO. 2015. Guideline on when to start antiretroviral therapy and on pre-exposure prophylaxis for HIV. Geneva, Switzerland: WHO.

${ }^{4}$ Fonner, V. A., et al. 2016. "Effectiveness and safety of oral HIV preexposure prophylaxis for all populations," AIDS 30(12): 1973.

${ }^{5}$ Van Damme, L. et al. 2012. "Preexposure prophylaxis for HIV infection among African women," New England Journal of Medicine 367(5): 411-422.

${ }^{6}$ Corneli, A. L. et al. 2014. "FEM-PrEP: adherence patterns and factors associated with adherence to a daily oral study product for pre-exposure prophylaxis." Journal of Acquired Immune Deficiency Syndromes 66(3): 324-331.

${ }^{7}$ World Health Organization. 2015. "Guideline on when to start antiretroviral therapy and on preexposure prophylaxis for HIV." Switzerland: World Health Organization.

${ }^{8}$ Mathur, S., N. Pilgrim, and J. Pulerwitz. 2016. "PrEP introduction for adolescent girls and young women," The Lancet HIV 3(9): e406-408.

${ }^{9}$ Castel, A. D. et al. 2015. “Understanding HIV care provider attitudes regarding intentions to prescribe PrEP," Journal of Acquired Immune Deficiency Syndromes 70(5): 520-528.

${ }^{10}$ Pilgrim, N. et al. 2016. "Building evidence to guide PrEP introduction for adolescent girls and young women." Washington, D.C.: Population Council

${ }^{11}$ Geibel, S. et al. 2017. "Stigma reduction training improves healthcare provider attitudes toward, and experiences of young marginalized people in Bangladesh," Journal of Adolescent Health 60(2): S35-44. 


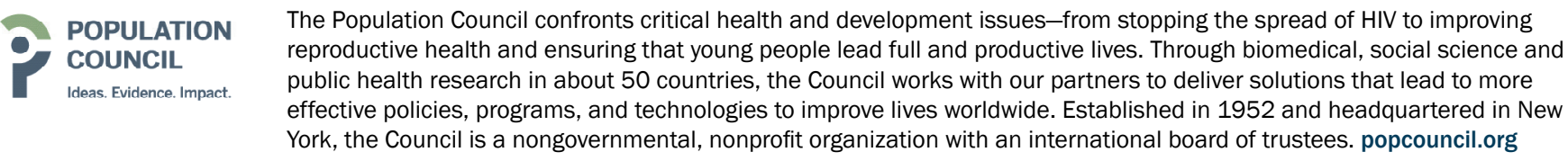

Suggested citation: Population Council. 2017. “Providers' views on PrEP for adolescent girls and young women in Tanzania: Findings from implementation science research," DREAMS Tanzania Results Brief 2. Washington, DC: Population Council. 